\title{
Serine/Threonine Protein Phosphatase 2A Catalytic Subunit Beta Isoform
}

National Cancer Institute

\section{Source}

National Cancer Institute. Serine/Threonine Protein Phosphatase 2A Catalytic Subunit

Beta Isoform. NCl Thesaurus. Code C34081.

Serine/threonine-protein phosphatase 2A catalytic subunit beta isoform (309 aa, 36

$\mathrm{kDa}$ ) is encoded by the human PPP2CB gene. This protein is involved in the dephosphorylation of proteins, the negative regulation of apoptosis and signal transduction. 